Aus der Vetsuisse-Fakultät Universität Zürich Klinik für Kleintiermedizin

Direktorin: Prof. Dr. Claudia Reusch

Arbeit unter Leitung von

Prof. Dr. Urs Giger

\title{
Radiographic Hip Joint Phenotype of the Pembroke Welsh Corgi
}

\author{
Inaugural-Dissertation \\ zur Erlangung der Doktorwürde der \\ Vetsuisse-Fakultät Universität Zürich
}

vorgelegt von

\section{Georga Tiffany Karbe}

Tierärztin

von Boynton Beach, Florida, USA

\section{1}

genehmigt auf Antrag von

Prof. Dr. Urs Giger, Referent

Zürich 2011 
Aus der Vetsuisse-Fakultät Universität Zürich Klinik für Kleintiermedizin

Direktorin: Prof. Dr. Claudia Reusch

Arbeit unter Leitung von

Prof. Dr. Urs Giger

\title{
Radiographic Hip Joint Phenotype of the Pembroke Welsh Corgi
}

\author{
Inaugural-Dissertation \\ zur Erlangung der Doktorwürde der \\ Vetsuisse-Fakultät Universität Zürich
}

vorgelegt von

\section{Georga Tiffany Karbe}

\author{
Tierärztin \\ von Boynton Beach, Florida, USA
}

2011

genehmigt auf Antrag von

Prof. Dr. Urs Giger, Referent

Zürich 2011 


\section{Table of Contents}

1 Summary 1

2 Introduction $\quad 2$

3 Materials and methods 3

3.1 Study population 3

3.2 Radiographic interpretation 3

3.3 Statistic analysis 3

$4 \quad$ Results 4

4.1 Distribution of age, weight, DI and joint abnormalities 4

4.2 Logistic regression analysis $\quad 5$

$\begin{array}{lll}4.3 & \text { Student t-test and z-test analysis } & 6\end{array}$

5 Discussion $\quad 6$

6 References 11

$7 \quad$ Curriculum Vitae 13

8 Acknowledgments 14 
Vetsuisse-Fakultät Universität Zürich (2011)

Georga T. Karbe

Department für Kleintiere, Vetsuisse-Fakultät Universität Zürich

msekey@vetclinics.uzh.ch

\section{Radiographic Hip Joint Phenotype of the Pembroke Welsh Corgi}

The purpose of this prospective and retrospective cross-sectional study was to assess the radiographic hip joint phenotype of the Pembroke Welsh Corgi. Coxofemoral joints of 399 Corgis were evaluated by the PennHIP method and standard ventrodorsal hip-extended radiographs for subluxation, osteoarthritis (OA), caudolateral curvilinear osteophytes (CCO), and circumferential femoral head osteophytes (CFHO). Joint laxity was measured by distraction index (DI). Few Corgis showed conventional OA $(6.8 \%)$ despite all Corgis having DI $>0.30 ; 18 \%$ had subluxation, $22.3 \%$ had CCO, and $74.4 \%$ had CFHO. Higher DI increased the odds for subluxation but not for OA, CCO or CFHO. The presence of CCO increased the odds for OA by 4.6 times $(\mathrm{p}=0.002)$ and 2.2 times $(\mathrm{p}=0.01)$ for hip dysplasia. All dogs with OA had CFHO. The presence of CFHO increased the odds for subluxation 8.7 times $(\mathrm{p}<0.001)$ and 8.9 times $(\mathrm{p}<0.001)$ for hip dysplasia. Subluxation increased the odds for OA by 15.4 times $(\mathrm{p}<0.001)$. Corgis showed a low frequency of conventional OA despite having joint laxity that has been shown to correlate with hip OA and hip dysplasia in large breed dogs. The relationship between $\mathrm{CCO}$ and $\mathrm{OA}$ was similar to published findings in non-chondrodystrophic, large breed dogs and the CFHO was significantly associated with hip dysplasia in this breed. The CCO is a sign of OA and the CFHO is a marker for subluxation; both are phenotypic-markers for hip dysplasia in this chondrodystrophic breed. The data suggests that the CFHO is part of the disease phenotype and not normal for the breed.

Keywords: hip dysplasia, osteoarthritis, degenerative joint disease, caudolateral curvilinear osteophyte, circumferential femoral head osteophyte. 


\section{Introduction}

Canine hip dysplasia is recognized as one of the most common and debilitating orthopedic diseases of large- and giant-breed dogs. ${ }^{1,2}$ It is a heritable disease of complex genetics, with defects in several yet to be identified genes. In addition to the genetic components, environmental factors, including diet, age, and body weight are known to influence the development of hip dysplasia and its clinical signs. ${ }^{3,4}$ Distinct differences in disease prevalence and susceptibility however, have been reported even among dogs of similar body size and weight: For instance German Shepherds are at a significantly greater risk for osteoarthritis (OA) than Golden retrievers, Labrador retrievers and Rottweilers having similar joint laxity. $1,5,6$

Joint laxity, evident as subluxation or measured by DI, is a well established risk factor for hip dysplasia in large-breed dogs. $3,4,7,8$ Osteophyte formations along the femoral neck, termed caudolateral curvilinear osteophytes (CCO) are important radiographic findings associated with hip dysplasia and can be considered early signs of OA. ${ }^{9-12}$ A distinct

Figure 1. Ventrodorsal, Hip-extended Radiograph of a 4.5 year old, female spayed Corgi with $\mathrm{CFHO}, \mathrm{CCO}$, subluxation and OA.

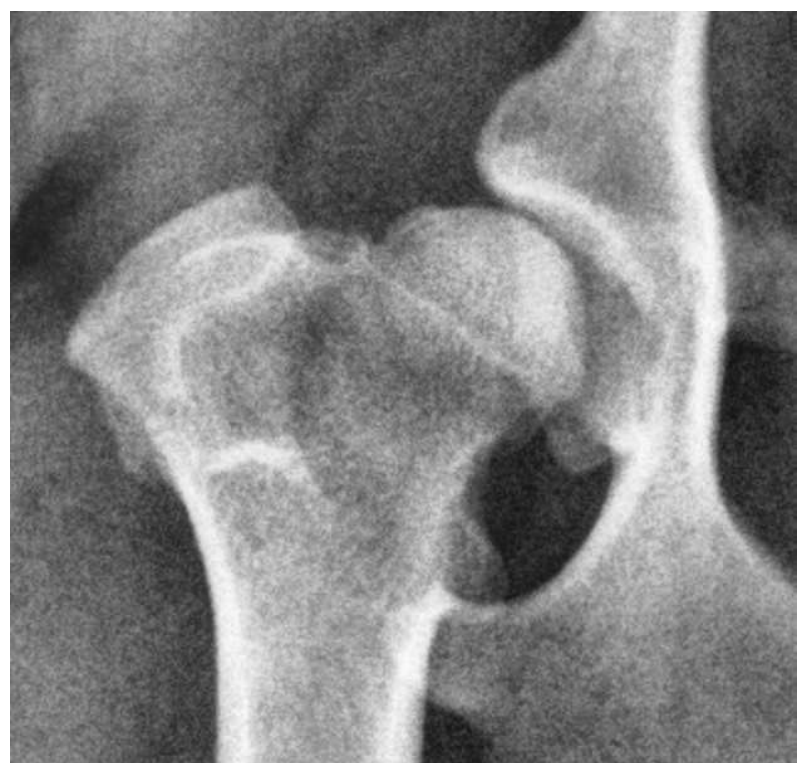

rim, appearing as a radiopaque line encircling the junction of the femoral head and neck at the site of capsular attachment, termed circumferential femoral head osteophyte (CFHO), has also been described as a sensitive predictor for the development and progression of OA in several large-breed dogs. ${ }^{12,13}$ This radiopaque rim was the earliest sign of hip dysplasia in a group of Labrador retrievers followed from birth until end of life. ${ }^{13}$ Both the $\mathrm{CCO}$ and $\mathrm{CFHO}$ have been associated with joint laxity and contribute to the diagnosis of hip dysplasia in large-breed dogs.

A distinct difference however, has been suggested between the CFHO in large-breed dogs and the presence of a radiopaque rim, similar in appearance and location, regularly found in adult chondrodystrophic dogs, particularly the Corgi. ${ }^{12,13}$ In these dogs the line represents a prominent, sharp rim encircling the femoral head, ${ }^{13}$ however, its importance and relationship to joint laxity, OA, or hip dysplasia have not been elucidated in chondrodystrophic breeds. Many radiologists consider this bony rim normal for the Corgi breed.

Although several small-breed dogs as well as cats, have been reported to develop hip dysplasia, little is currently known about the genetic predisposition, risk factors, disease progression, or clinical and radiographic features in these breeds and species. ${ }^{2,14-19}$ In one registry (Orthopedic Foundation for Animals [OFA]) the Pug is listed as having the second highest prevalence of hip dysplasia $(63.8 \%)$ and several other small-breed dogs seem to be commonly affected. ${ }^{2}$

It was the purpose of this investigation to describe the radiographic hip joint phenotype of the Pembroke Welsh Corgi and to test two diametrically opposed hypotheses: that $\mathrm{CFHO}$ has no relationship to hip dysplasia and is therefore normal for the breed, or alternatively, that $\mathrm{CFHO}$ is a radiographic marker, riskfactor, or osteophytic component of canine hip dysplasia in the Corgi breed just as it is in nonchondrodystrophic dogs. 


\section{Materials and Methods}

Hip radiographs derived from two sample populations of Pembroke Welsh Corgis were included in this study: (1) Archived ventrodorsal, hip-extended radiographs, and compression and distraction radiographs of Corgis drawn from the PennHIP database, and (2) Prospectively acquired hip-extended, compression, and distraction hip radiographs from Corgis, $\geq 4$ years of age recruited for this study. The study was approved by the Institutional Animal Care and Use Committee with a Patient-owned Animal Protocol and client informed consent.

All $\mathrm{HE}$ radiographs were digitized ${ }^{20}$ and evaluated by the same board-certified radiologist for signs of hip dysplasia, OA, subluxation, $\mathrm{CCO}$, and $\mathrm{CFHO}$. The $\mathrm{CCO}$ was visible as a radiopaque line located on the caudal aspect of the femoral neck as seen on the HE radiograph. ${ }^{9-11,21}$ The presence of a CFHO was subjectively determined based on the appearance of a radiopaque rim encircling the junction of the femoral head and neck near the site of capsular attachment (Figure 1). ${ }^{13}$ The diagnosis of OA followed conventional criteria, based on the appearance of subchondral bone sclerosis along the craniodorsal acetabulum and/or osteophytes on the cranial and caudal aspects of the acetabular margin or femoral

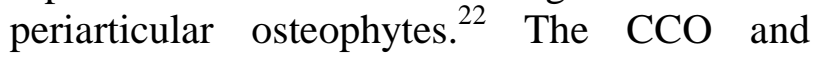
CFHO were evaluated separately and were not factors included in the diagnosis of OA or hip dysplasia. Hip-extended radiographs were subjectively graded as dysplastic or normal based on an OFA-type scoring system. Subjectively-determined subluxation without radiographic signs of OA was graded as mild hip dysplasia. ${ }^{2}$ Hip joints showing osteoarthritis with or without evidence of subluxation were scored as moderate or severe hip dysplasia based on the progression of OA.

Each dog is represented once within this study. All dogs were included if the respective hip films were available in the archives. For statistical analyses, dogs were included if a DI was recorded for at least one hip joint. The larger (looser) DI was chosen to represent an objective measure of hip joint laxity for each dog. Seven dogs in this study had DI available from only one hip due to the presence of cavitation $^{23}$ on the opposite hip. In these cases, the single DI was chosen to represent the dog. The existence of $\mathrm{OA}, \mathrm{CCO}, \mathrm{CFHO}$ and subluxation from either or both hips were recorded and used in the analyses. Comparisons of mean age, body weight and DI between disease groups were performed using the Student's t-test. Proportions of joint abnormalities were compared using the z-test. For the purposes of the study Corgis were grouped into 3 age intervals: 1) $4-11$ months (juveniles), 2) 12 - 23 months (young adults) and 3) $\geq 24$ months (adults). A one-way ANOVA was performed to evaluate differences in mean DI and mean weight among these age groups. To assess the prevalence of radiographic findings by age, Corgis were divided into the following age groups: $<0.5$; $\geq 0.5$ to $<1 ; \geq 1$ to $<2 ; \geq 2$ to $<4 ; \geq 4$ to $<6$; and $\geq 6$ years. Based on the age groups the relationships between age and prevalence of subluxation, $\mathrm{OA}, \mathrm{CCO}$ and $\mathrm{CFHO}$ were evaluated. Five separate logistic regression models were constructed with the dependent variable being hip dysplasia, subluxation, OA, $\mathrm{CCO}$ and $\mathrm{CFHO}$. The logistic regression analyses were used to determine the effect of covariates; age, weight, gender, DI, subluxation, OA, $\mathrm{CCO}$ and $\mathrm{CFHO}$ as risk factors for the radiographic existence of each of these dependent variables (excluding the respective outcome variable). Odds ratios (OR) were calculated from the logistic model for significant covariates (risk factors). All tests were performed using commercially available statistical software (SPSS 12.0 for windows, Copyright SPSS 1989-2003. Chicago, Illinois). Statistical significance was set at $\mathrm{p}<0.05$. 


\section{Results}

A total of 399 Pembroke Welsh Corgis were evaluated radiographically for hip dysplasia in this study; 372 were retrospective cases from the archives, radiographed between 1995 and 2010, and 27 older Corgis ( $\geq 4$ years of age) were prospectively examined in 2009. Ranges and means of age, weight, and DI as well as prevalence of hip dysplasia, OA, CCO, $\mathrm{CFHO}$, and subluxation were tabulated (Table $1)$.

Logistic regression analysis showed age to be a significant risk factor for hip dysplasia, $\mathrm{OA}, \mathrm{CCO}$ and $\mathrm{CFHO}$; for every 1 year increase in age, the odds for hip dysplasia increased 1.3 times $(p=0.002)$, the odds for OA increased 1.5 times $(p<0.001)$ and the odds for CCO and CFHO increased 1.4 times $(\mathrm{p}<0.001 ; \mathrm{p}=0.004$ respectively) (Table 2 ). The probability for OA increased for dogs having contemporaneous coxofemoral subluxation with an odds ratio of 15.4 ( $\mathrm{p}<0.001)$. The prevalence of OA increased linearly with age $\left(r^{2}=0.945 ; p=0.001\right)$ (Figure 2).

Dogs with CCO were significantly older and heavier than those without CCO (student ttest; $\mathrm{p}<0.001$ and $\mathrm{p}<0.001$ respectively). The presence of $\mathrm{CCO}$ significantly increased the odds for hip dysplasia (OR 2.2, $\mathrm{p}=0.01$ ) and OA (OR 4.6, $\mathrm{p}=0.002$ ) but did not significantly increase the odds for subluxation (Table 2). The prevalence of CCO increased linearly with age $\left(r^{2}=0.976 ; p<0.001\right)$ (Figure 2).

All Corgis with conventionally determined OA had a CFHO. Using a student ttest, Corgis with radiographic evidence of $\mathrm{CFHO}$ were found to be significantly heavier $(p<0.001)$ and older $(p<0.001)$ than dogs without this radiographic feature (Table 3). For every $1 \mathrm{~kg}$ increase in body weight the odds for CFHO increased by 1.5 times $(\mathrm{p}<0.001)$. Dogs with CFHO had 8.7 times $(p=0.004)$ greater odds for having subjective subluxation on $\mathrm{HE}$ radiographs and 8.9 times $(\mathrm{p}<0.001)$ greater odds for conventional hip dysplasia (Table 2). A linear increase of CFHO prevalence was seen in Corgis between 4 months and 1 year of age, after which the $\mathrm{CFHO}$ prevalence remained constant at $>80 \%$ for all age groups older than 1 year of age (Table 4, Figure 2).

Based on the presence of conventional OA and/or subluxation, 20\% (80/399) of the Corgis were considered dysplastic, of which $90 \%(72 / 80)$ had coxofemoral subluxation and $34 \%(27 / 80)$ had OA. Only one dog younger

Table 1. Distribution of Age, Weight, DI, and Joint Abnormalities

\begin{tabular}{|c|c|ccc|cc|}
\hline & All & Juvenile & Young Adult & Adult & Females & Males \\
\hline $\mathbf{n}$ & $399^{\ddagger}$ & 89 & 113 & 197 & 247 & 152 \\
Age $^{*}$ & $2.3 \pm 1.8$ & 0.62 & 1.4 & 3.6 & 2.29 & 2.41 \\
(years) $^{*}$ & $(0.31-10.79)$ & $(0.31-0.99)$ & $(1.0-1.99)$ & $(2.0-10.8)$ & $(0.31-10.6)$ & $(0.33-10.8)$ \\
Weight $^{* \dagger}$ & $11.3 \pm 2.5$ & 9.0 & 11.6 & 12.2 & 10.72 & 12.37 \\
(kg) & $(5.0-23.6)$ & $(5.0-1145)$ & $(7.7-16.8)$ & $(7.7-23.6)$ & $(5.0-23.6)$ & $(5.0-17.7)$ \\
DI* & $0.66 \pm 0.13$ & 0.68 & 0.65 & 0.66 & 0.68 & 0.64 \\
OA (\%) & $(0.33-1.06)$ & $(0.36-1.0)$ & $0.39-0.95)$ & $(0.33-1.06)$ & $(0.35-1.06)$ & $(0.33-0.97)$ \\
CCO (\%) & 6.8 & 1.1 & 0 & 13.2 & 7.3 & 5.9 \\
CFHO (\%) & 22.3 & 5.6 & 17.7 & 32.5 & 7.3 & 5.9 \\
Sblx (\%) & 74.4 & 29.2 & 85.8 & 88.3 & 21.5 & 23.7 \\
CHD (\%) & 18.0 & 7.9 & 9.7 & 27.4 & 74.9 & 73.7 \\
\hline
\end{tabular}

*Mean \pm SD (range); †overall weight based on 368 dogs; $\ddagger$ n: number of dogs.

Juvenile: dogs age < 12 months; Young Adult: dogs age 12-23 months; Adult: dogs $\geq 24$ mths 
Table 2. Logistic Regression Analyses: Dependent variables are hip dysplasia (CHD), subluxation, OA, $\mathrm{CCO}$ and $\mathrm{CFHO}$. Covariates are age, weight, gender DI, subluxation, CCO, CFHO, OA (only statistically significant covariates listed with $\mathrm{p}<0.05)$.

\begin{tabular}{|c|c|c|c|}
\hline \multicolumn{4}{|c|}{ Dependent variable $=$ Subluxation } \\
\hline $\begin{array}{c}\text { Significant } \\
\text { Covariates }\end{array}$ & $\begin{array}{c}\text { Value } \\
(\mathrm{B})\end{array}$ & Sig. & OR* \\
\hline DI & 7.921 & $<0.001$ & 2753.865 \\
\hline OA & 2.438 & $<0.001$ & 11.446 \\
\hline CFHO & 2.163 & $<0.001$ & 8.694 \\
\hline
\end{tabular}

* Odds ratio (OR) for each 0.10 increase in DI is 2.21

\begin{tabular}{|c|c|c|c|}
\hline \multicolumn{4}{|c|}{ Dependent variable $=$ Hip Dysplasia $($ CHD) } \\
\hline $\begin{array}{c}\text { Significant } \\
\text { Covariates }\end{array}$ & $\begin{array}{c}\text { Value } \\
(\mathrm{B})\end{array}$ & Sig. & OR $^{*}$ \\
\hline DI & 7.485 & $<0.001$ & 1781.738 \\
\hline Age (Y) & 0.243 & 0.002 & 1.275 \\
\hline CCO & 0.799 & 0.010 & 2.222 \\
\hline CFHO & 2.187 & $<0.001$ & 8.910 \\
\hline
\end{tabular}

* OR for each 0.10 increase in DI is 2.11

\begin{tabular}{|c|c|c|c|}
\hline \multicolumn{4}{|c|}{ Dependent variable $=$ OA } \\
\hline $\begin{array}{c}\text { Significant } \\
\text { Covariates }\end{array}$ & $\begin{array}{c}\text { Value } \\
(\mathrm{B})\end{array}$ & Sig. & OR \\
\hline Age $(\mathrm{Y})$ & 0.405 & $<0.001$ & 1.499 \\
\hline CCO & 1.529 & 0.002 & 4.612 \\
\hline Subluxation & 2.737 & $<0.001$ & 15.446 \\
\hline
\end{tabular}

\begin{tabular}{|c|c|c|c|}
\hline \multicolumn{4}{|c|}{ Dependent variable $=$ CCO } \\
\hline $\begin{array}{c}\text { Significant } \\
\text { Covariates }\end{array}$ & $\begin{array}{c}\text { Value } \\
(\mathrm{B})\end{array}$ & Sig. & OR \\
\hline Age (Y) & 0.069 & $<0.001$ & 1.358 \\
\hline OA & 0.448 & $<0.001$ & 5.509 \\
\hline
\end{tabular}

\begin{tabular}{|c|c|c|c|}
\hline \multicolumn{4}{|c|}{ Dependent variable $=$ CFHO } \\
\hline $\begin{array}{c}\text { Significant } \\
\text { Covariates }\end{array}$ & $\begin{array}{c}\text { Value } \\
(\mathrm{B})\end{array}$ & Sig. & OR \\
\hline Age $(\mathrm{Y})$ & 0.316 & 0.004 & 1.371 \\
\hline Weight $(\mathrm{Kg})$ & 0.382 & $<0.001$ & 1.465 \\
\hline Gender & 0.624 & 0.048 & 1.867 \\
\hline Subluxation & 2.147 & 0.004 & 8.562 \\
\hline
\end{tabular}

than 1 year of age was found to have OA, whereas the remaining dogs in this age group were diagnosed to have hip dysplasia based on the presence of subluxation alone. DI significantly increased the odds for subluxation; every 0.1 increase in DI increased the odds for subluxation by 2.2 times $(\mathrm{p}<0.001)$ (Table 2). Similarly the mean DI of Corgis considered dysplastic (0.74) was significantly higher than the mean DI of non-dysplastic (0.65) Corgis (student t-test; $\mathrm{p}<0.001$ ) (Table $3)$. For every 0.1 increase in DI the odds for hip dysplasia, conventionally diagnosed on $\mathrm{HE}$ radiographs, increased by 2.1 times $(\mathrm{p}<0.001)$.

Radiographic lines on the proximal femur (CCO or CFHO) were frequently seen in dysplastic Corgis with $95 \%$ (76/80) having CFHO and $40 \%(32 / 80)$ CCO. Of the Corgis older than 2 year of age, $31 \%$ (61/197) were considered dysplastic, with $89 \%$ (54/61) having subluxation and $43 \%$ (26/61) having OA. Proximal femoral radiopaque lines were also frequently seen in dysplastic Corgis with $98 \%$ (60/61) having CFHO and 49\% (30/61) having CCO. These radiographic findings were, however, also found in non-dysplastic dogs in this age group, $84 \%(114 / 136)$ and $25 \%$ (34/136) had CFHO and CCO, respectively.

Significant differences in hip laxity (DI) and weight were seen between male and female Corgis. Male Corgis were significantly (student t-test; $\mathrm{p}<0.001$ ) heavier than female Corgis whereas females had significantly looser DI than males (Student t-test; $\mathrm{p}=0.01$ ). Using logistic regression analysis, female Corgis had 1.9 times greater odds for having $\mathrm{CFHO}$ than males $(\mathrm{p}=0.048)$. Gender status (intact vs. spayed/neutered) was recorded for $166 \mathrm{dogs}$; 17 were spayed females, 86 intact females, 17 neutered males and 46 intact males. 
Table 3a. Radiographic Joint Abnormalities.

\begin{tabular}{|c|c|c|c|c|c|c|c|c|c|c|c|c|c|c|c|}
\hline & OA & $\mathbf{O A}$ & $\mathbf{P}$ & $\mathrm{CCO}$ & $\mathrm{CCO}$ & $\mathbf{P}$ & CFHC & $\overline{\mathrm{CFHO}}$ & $\mathbf{P}$ & $\begin{array}{c}\text { Sblx } \\
+\end{array}$ & $\begin{array}{c}\text { Sblx } \\
-\end{array}$ & $\mathbf{P}$ & $\begin{array}{c}\text { CHD } \\
+\end{array}$ & $\begin{array}{c}\text { CHD } \\
-\end{array}$ & $\mathbf{P}$ \\
\hline$n$ & 27 & 372 & & 89 & 310 & & 297 & 102 & & 72 & 327 & & 80 & 319 & \\
\hline Age & 4.1 & 2.2 & $<0.001$ & 3.3 & 2 & $<0.001$ & 2.7 & 1.4 & $<0.001$ & 2.8 & 2.2 & 0.014 & 3.1 & 2.1 & $<0.001$ \\
\hline DI & 0.7 & 0.66 & $\mathrm{~ns}$ & 0.67 & 0.66 & ns & 0.66 & 0.68 & ns & 0.73 & 0.65 & $<0.001$ & 0.74 & 0.65 & $<0.001$ \\
\hline Weight & 12.4 & 11.3 & 0.011 & 12.1 & 11.1 & $<0.001$ & 11.9 & 9.7 & $<0.001$ & 12.2 & 11.2 & $<0.001$ & 12.3 & 11.1 & $<0.001$ \\
\hline
\end{tabular}

Statistical difference between mean age, weight and DI calculated using Student t-test. (+ present, - absent)

*overall weight based on 368 dogs. Sblx: coxofemoral subluxation; CHD: canine hip dysplasia

Table 3b. Radiographic Joint Abnormalities.

\begin{tabular}{|c|c|c|c|c|c|c|c|c|c|c|c|c|c|c|c|}
\hline \multirow{2}{*}{\multicolumn{2}{|c|}{$\begin{array}{c}\text { OA } \\
+ \\
\end{array}$}} & \multirow{2}{*}{$\begin{array}{c}\text { OA } \\
- \\
\end{array}$} & \multirow[t]{2}{*}{$\mathbf{P}$} & \multirow{2}{*}{$\begin{array}{c}\mathrm{CCO} \\
+ \\
\end{array}$} & \multirow{2}{*}{$\begin{array}{c}\mathrm{CCO} \\
- \\
\end{array}$} & \multirow[t]{2}{*}{$\mathbf{P}$} & \multicolumn{2}{|c|}{ CFHO CFHO } & \multirow[t]{2}{*}{$\mathbf{P}$} & \multirow{2}{*}{$\begin{array}{c}\text { Sblx } \\
+ \\
\end{array}$} & \multirow{2}{*}{$\begin{array}{c}\text { Sblx } \\
- \\
\end{array}$} & \multirow[t]{2}{*}{$\mathbf{P}$} & \multirow{2}{*}{$\begin{array}{c}\text { CHD } \\
+ \\
\end{array}$} & \multirow{2}{*}{$\begin{array}{c}\text { CHD } \\
- \\
\end{array}$} & \multirow[t]{2}{*}{$\mathbf{P}$} \\
\hline & & & & & & & + & - & & & & & & & \\
\hline$n$ & 27 & 372 & & 89 & 310 & & 297 & 102 & & 72 & 327 & & 80 & 319 & \\
\hline $\mathbf{O A}$ & - & - & - & $20 \%$ & $3 \%$ & $<0.001$ & $9 \%$ & $0 \%$ & 0.0 & $26 \%$ & $2 \%$ & $<0.001$ & $34 \%$ & $0 \%$ & $<0.001$ \\
\hline $\mathrm{CCO}$ & $67 \%$ & $19 \%$ & $<0$. & - & - & - & $26 \%$ & $11 \%$ & 0.0 & $38 \%$ & $19 \%$ & $<0$ & $40 \%$ & $18 \%$ & $<0.001$ \\
\hline CFHO & $100 \%$ & $73 \%$ & 0.002 & $88 \%$ & $71 \%$ & 0.001 & - & - & - & $94 \%$ & $70 \%$ & $<0.001$ & $95 \%$ & $69 \%$ & $<0.001$ \\
\hline Sblx & $70 \%$ & $14 \%$ & $<0.001$ & $30 \%$ & $15 \%$ & $<0.001$ & $23 \%$ & $4 \%$ & $<0.001$ & - & - & - & $90 \%$ & $0 \%$ & $<0.001$ \\
\hline
\end{tabular}

Statistical differences in proportions of joint abnormalities calculated using z-test. (+ present, - absent).

\section{Discussion}

While hip dysplasia has been clinically and radiographically well characterized in several large- and giant-breed dogs, to the authors' knowledge this is the first study focusing on the hip joints of a small chondrodystrophic breed. The Pembroke Welsh Corgi was selected, because of the suggested unique hip joint conformation, including joint laxity, $\mathrm{CCO}$, and $\mathrm{CFHO}$ and their unstudied relationship with hip dysplasia. According to the PennHIP database, the Pembroke Welsh Corgi with a mean DI of 0.66 , is, as of March 2011 , one of the 10 breeds having highest mean hip laxity. ${ }^{24}$ In the present study, the radiographic hip joint phenotype of this breed was associated with a high degree of joint laxity based on DI and subluxation, the frequent appearance of periarticular, radiopaque lines, and rarely with signs of conventional OA. All 399 Corgis radiographically examined had hip laxity in the disease-susceptible range, (DI $\geq 0.30)^{25}$ with higher (looser) DI significantly increasing the odds for conventional hip dysplasia, consistent with findings in large breed dogs. ${ }^{5,7}$ While the degree of joint laxity $(0.66 \pm 0.13$; mean DI \pm SD) would have predicted an average $20-60 \%$ probability for the development of OA in largebreed dogs older than 2 years of age, ${ }^{5}$ only $13.2 \%$ of Corgis older than 2 years manifested radiographic findings of conventionally defined OA. Other osteophyte-like radiographic features, however, were highly prevalent in this group of Corgis. The CCO was associated with hip disease; its presence significantly increasing the odds for OA and hip dysplasia. Similarly the CFHO significantly increased the odds for hip dysplasia and subluxation, but not $\mathrm{OA}$. The CCO and CFHO were associated with different components of hip dysplasia, namely osteoarthritis and subluxation, respectively so that each radiographic feature represents a component of hip dysplasia in the Corgi.

Joint laxity by DI indicated that all members of this sample of Corgis were OA susceptible, with higher DI increasing the odds for hip dysplasia. The absence of dogs with DI 
below 0.30 made it impossible to determine a definitive relationship, between these radiopaque lines and the full clinical range of DI. Interestingly, female Corgis were at a greater risk for having a CFHO than males, with females $(0.68)$ having significantly higher DI than males (0.64). The gender difference in DI of 0.04 is small and may not have clinical significance, however, it is the first breed in which it has been found. Although speculation, it may reflect greater selection pressure for better hips in males or, alternatively, it may represent greater selection bias for males to have PennHIP evaluation.

The radiographic hip joint phenotype suggests that despite severe hip laxity, only a small proportion of Corgis show characteristic radiographic evidence of $\mathrm{OA}$ and the breed therefore may be less susceptible to hip dysplasia. The OFA ranks the Pembroke Welsh Corgi as one of the top 50 breeds in terms of hip dysplasia prevalence $(18.4 \%$ as of May 2011). ${ }^{2}$ Similarly the present study diagnosed hip dysplasia in $31 \%$ of Corgis older than 2 years of age, agreeing favorably with this figure. The lack of conventional radiographic signs of hip OA in this breed motivated us to scrutinize the hips for other phenotypic signs of disease, such as coxofemoral subluxation or non-conventional

radiographic features such as the $\mathrm{CCO}$ and $\mathrm{CFHO}$ to determine their possible role in disease susceptibility. Of the dysplastic dogs older than 2 year of age, $89 \%$ (54/61) were diagnosed dysplastic based on the radiographic appearance of subluxation which suggests that joint laxity in the Corgi is an important marker of disease, though not by itself a definitive sign of a degenerative process.

Subluxation as evident on the hipextended radiograph has empirically been accepted to be the primary cause of hip OA in dysplastic dogs. ${ }^{8}$ To date there are no definitive studies evaluating the relationship between subluxation and the development of OA despite its worldwide acceptance as a hallmark for disease. The DI in comparison has repeatedly been shown to be a significant risk factor for OA in large-breed dogs. ${ }^{3,4}$ In this cohort of Corgis only $26 \%(19 / 72)$ of those with subluxation on hip-extended radiographs had conventional OA whereas 94\% (68/72) had a CFHO. Similarly, only $8 \%$ (27/399) of Corgis
Figure 2. Proportion of Radiographic Findings by Age Groups

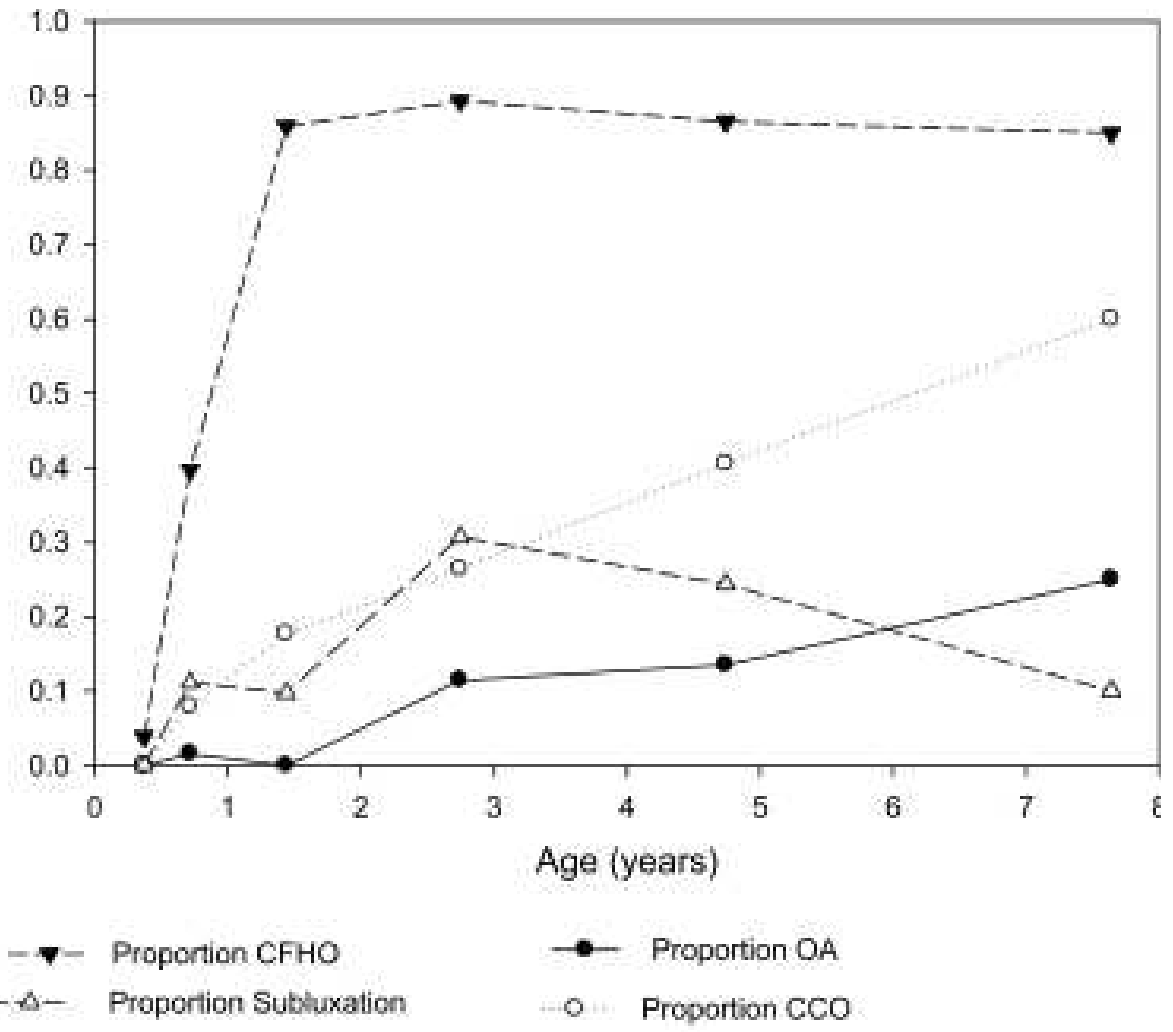

Plots on graph represent proportion of OA, subluxation, $\mathrm{CFHO}$ and $\mathrm{CCO}$ at the mean age for each age group ( 0.37 years; 0.72 years; 1.44 years; 2.75 years; 4.74 years; 7.64 years). Age groups represented in Table 4. 
with DI in the OA-susceptible range ( $\mathrm{DI} \geq 0.30$ ) had OA whereas $74.4 \%$ (297/399) had a CFHO. All dogs with subluxation were considered dysplastic, in this group of Corgis however only a small percentage with joint laxity by subluxation had radiographic evidence of conventional OA, whereas a vast majority had a CFHO. The appearance of radiographic $\mathrm{OA}$ is accepted to be agedependent and its progression is influenced by environmental factors such as body weight. ${ }^{3,4,22}$ In this group of young Corgis it may be argued that the breed's small body size and young average age contributed to the low OA prevalence despite presence of subluxation and high DI measurements. In contrast, the CFHO was highly prevalent and radiographically manifested at 1 year of age. The significant relationship between $\mathrm{CFHO}$ and subluxation and hip dysplasia support the hypothesis that this radiopaque rim is a component of hip dysplasia in the Corgi and may even be the earliest radiographic indicator.

In large-breed dogs, CFHO was found to be the earliest radiographic finding in 50 to $70 \%$ of dogs that subsequently developed hip dysplasia and was present in $87 \%$ of dogs 6-7 months of age that went on to develop OA by 2 years of age. ${ }^{12,13}$ Although dogs were not followed longitudinally in the present study, all Corgis were disease-susceptible based on the described DI threshold and the presence of CFHO was significantly linked to subluxation as seen on the hip-extended radiograph. A marked increase in CFHO prevalence was observed between dogs younger than 1 year of age and those older than 1 year of age (Figure 2), suggesting that $\mathrm{CFHO}$ is a progressive, likely degenerative, phenomenon like other signs of hip dysplasia. A similar rapid increase in CFHO prevalence between 6 months and 1 year of age was reported in a group of Foxhounds, of which the vast majority ultimately developed OA. ${ }^{12}$ It was suggested that the Foxhound's severe hip laxity led to this rapid CFHO development. In the Corgi the presence of $\mathrm{CFHO}$ was significantly associated with subluxation and on average, the Corgis' hip laxity was similarly severe as that of the Foxhound so that the CFHO in the Corgi may also be laxity-based and, therefore, also a part of the hip dysplasia complex. In contrast to the Foxhounds, Corgis manifested little radiographic signs of conventional OA despite similar degrees of joint laxity (DI). This discrepancy may be attributed to the marked difference in body weight between Corgis $(12 \mathrm{~kg})$ and Foxhounds $(30 \mathrm{~kg})$, the chondrodystrophic conformation and other contributing factors yet undetermined, which may act to delay or prevent development of conventional OA. Although the etiology and pathogenesis of

Table 4. Proportions of OA, subluxation, $\mathrm{CCO}, \mathrm{CFHO}$ and hip dysplasia by Age Group

\begin{tabular}{|c|c|c|c|c|c|c|c|c|}
\hline $\begin{array}{c}\text { Age } \\
\text { Group } \\
\text { (years) }\end{array}$ & $\mathbf{n}^{*}$ & $\begin{array}{c}\text { Mean } \\
\text { weight } \\
(\mathbf{k g})^{\dagger}\end{array}$ & $\begin{array}{c}\text { Mean } \\
\text { DI }\end{array}$ & OA & Sblx & CCO & CFHO & CHD \\
\hline$<0.5$ & 26 & 67 & 0.72 & $0(-)$ & $0(-)$ & $0(-)$ & $1(4)$ & $0(-)$ \\
\hline$\geq 0.5-<1$ & 63 & 10.1 & 0 . & $1(2)$ & 7 & 5 & 25 & $8(13)$ \\
\hline$\geq 1-<2$ & 113 & 11.6 & 0.65 & $0(-)$ & 11( & 20( & 97 & $11(10)$ \\
\hline$\geq 2-<4$ & 140 & 12.1 & 0.67 & $16(11)$ & $43(31)$ & 37 (26) & $125(89)$ & $45(32)$ \\
\hline$\geq 4-<6$ & 37 & 12.5 & 0.65 & $5(14)$ & $9(24)$ & $15(41)$ & $32(86)$ & $10(27)$ \\
\hline$\geq 6$ & 20 & 12.5 & 0.59 & $5(25)$ & $2(10)$ & $12(60)$ & $17(85)$ & $6(30)$ \\
\hline
\end{tabular}

$* n$ : number of dogs in each age group. ${ }^{\dagger}$ over all weights based on $n=368$

The proportions of OA, CCO, and CFHO are based on appearance in either right or left hip joint or both $[\mathrm{n}(\%)]$. Mean DI calculated using highest DI per dog.

See Figure 2 this radiopaque rim are still not completely understood, its presence in the Corgi was significantly associated with subluxation which in itself is an accepted phenotype of hip dysplasia. 
Hip Joint Phenotype of the Pembroke Welsh Corgi

Table 5. Proportions of OA, subluxation, CCO, CFHO and OFA grades by DI intervals

\begin{tabular}{|c|c|c|c|c|c|c|c|c|}
\hline DI Interval & $\mathbf{N}$ & $\begin{array}{c}\text { Mean } \\
\text { Age }\end{array}$ & $\begin{array}{c}\text { Mean } \\
\text { Weight }^{\dagger}\end{array}$ & OA & CCO & CFHO & Sblx & OFA grades* \\
\hline $0.33-0.49$ & 53 & 3.1 & 12.0 & $2(3.8)$ & $10(18.9)$ & $41(77.4)$ & $\begin{array}{c}0 \\
(-)\end{array}$ & $\begin{array}{c}51 \text { good } \\
1 \text { moderate } \\
1 \text { severe }\end{array}$ \\
\hline $0.50-0.59$ & 127 & 2.2 & 11.3 & $4(3.1)$ & $26(20.5)$ & $96(75.6)$ & $12(9.4)$ & $\begin{array}{l}113 \text { good } \\
1 \text { fair } \\
9 \text { mild } \\
4 \text { moderate }\end{array}$ \\
\hline $0.60-0.69$ & 108 & 2.4 & 11.4 & $10(9.3)$ & $32(29.6)$ & $81(75.0)$ & $25(23.1)$ & $\begin{array}{c}80 \text { good } \\
18 \text { mild } \\
8 \text { moderate } \\
2 \text { severe }\end{array}$ \\
\hline$\geq 0.70$ & 111 & 2.0 & 11.1 & $11(9.9)$ & $21(18.9)$ & $79(71.2)$ & $35(31.5)$ & $\begin{array}{c}73 \text { good } \\
1 \text { fair } \\
26 \text { mild } \\
9 \text { moderate } \\
2 \text { severe }\end{array}$ \\
\hline
\end{tabular}

"OFA grades: OFA-type grades assigned by the radiologist; ${ }^{\dagger}$ over all weights based on $n=368$; The proportions of $\mathrm{OA}, \mathrm{CCO}$, and CFHO are based on appearance in either right or left hip joint or both [n(\%)]. Mean DI calculated using highest DI per dog.

While body weight is a well-recognized non-genetic factor that influences the manifestation and progression of hip dysplasia, ${ }^{3,4}$ OA and hip dysplasia are historically reported to be rare $(0.2 \%)$ in dogs weighing $<9 \mathrm{~kg} .{ }^{26}$ Similarly, no OA was seen in Corgis in this sample weighing $<10 \mathrm{~kg}$ and dogs without a CFHO on average weighed 9.7 $\mathrm{kg}$. The only Corgi younger than 1 year of age found to have conventional $\mathrm{OA}$ weighed $12.7 \mathrm{~kg}$. Although the overall average Corgi weighed $>9 \mathrm{~kg}$, their weight may still be low enough to contribute to a higher laxity tolerance, lower disease susceptibility and slower disease progression. It is important to note, however, that Corgis weighing $<10 \mathrm{~kg}$ were on average significantly younger (1.3 years) than those weighing $\geq 10 \mathrm{~kg}$ (2.7 years) so perhaps degenerative radiographic joint changes had not yet developed in the lighter dogs.

It may be speculated from this sample of Corgis that the described threshold of 0.30 for large-breed dogs does not apply to the Corgi breed and that perhaps a higher (looser) DI should be considered when evaluating small, chondrodystrophic breeds. In the present study however, radiographic OA was found in Corgis with DIs as low as 0.37 and 0.47 , which is suggestive that a similar OA threshold applies to Corgis as it does to nonchondrodystrophic dogs. Minimally, the present data does not refute the previously described 0.30 threshold in the Corgi breed. In this large sample of 399 Corgis there were no dogs with DI below 0.30; therefore, it could not be definitively assessed whether Corgis with tight hips would show radiographic OA. This represents a limitation of the study population; however, it was unavoidable given the comparatively high hip laxity in the Corgi breed as a whole.

Passive laxity as measured by DI, has been shown to put dogs at risk for functional laxity, creating abnormal forces in the weightbearing hip and ultimately resulting in joint pathology. ${ }^{6}$ Laxity tolerance in the Corgi may have reduced the development of conventional radiographic signs of $\mathrm{OA}$, but not the formation of osteophyte-like, radiopaque lines such as the $\mathrm{CCO}$ and $\mathrm{CFHO}$, making them perhaps diagnostically useful. Subluxation during weight-bearing places abnormal, forces on the articular cartilage, leading to joint 
inflammation and ultimately thickening of the joint capsule and bony spurs at sites of capsular attachment to the bone. ${ }^{9,27}$ It is conceivable that the manifestations of severe joint laxity in the Corgi, may be different than the conventional degenerative process observed in large breed dogs and instead it may manifest radiographically as $\mathrm{CCO}$ or $\mathrm{CFHO}$. If true, and if these radiographic features are not included as diagnostic signs of hip dysplasia, the vast majority of dogs approved for breeding may, in fact, be dysplastic. The significance of the nonconventional osteophytes for diagnosing hip dysplasia in the Corgi, though not identical, is comparable to reports from large-breed dogs.

Due to the large proportion of Corgis manifesting this radiographic sign it has empirically been deemed 'normal' for the breed. The results of this study question this belief since all Corgis had DI in the diseasesusceptible range and the CFHO was significantly associated with subluxation and hip dysplasia. Although perhaps considered 'normal' for the breed the CFHO (and hip dysplasia) like chondrodysplasia itself, may be fixed within the breed. Disproportional dwarfism is anaccepted genetic trait in the Corgi, and therefore considered desirable. ${ }^{28}$ Hip dysplasia on the other hand, may have similar ubiquity but is distinctly undesirable as a genetic trait within the breed. In order to reduce the frequency of a multifactorial genetic disease such as hip dysplasia, appropriate selection pressure must be applied to the breeding population. ${ }^{29-31}$ The CFHO in addition to the $\mathrm{CCO}$ and other accepted phenotypic signs of hip dysplasia should be taken into account when selecting genetically desirable breeding animals. It is important to emphasize that we are not by any means suggesting that all dogs with CFHO at 2 years of age be excluded from breeding. This would limit the breeding pool to only $12 \%$ of the breed and likely create deleterious genetic bottlenecks. Rather, based on the principles of quantitative genetics, Corgi breeders are encouraged to submit dogs for
PennHIP evaluation andselect breeding candidates from the tighter half (tighter than breed average DI) of the population thereby reducing hip laxity from one generation to the next. Following these time-tested principles of quantitative genetics ${ }^{30}$, the prevalence of CCO and $\mathrm{CFHO}$ (and hip dysplasia) will be methodically lowered. However, given the Corgi's high hip laxity distribution, it is unclear whether the breed average can be moved to less than a DI of 0.30 even after many generations of selection; unless strategies like outcrossing would be implemented.

In contrast to the radiographic onset of $\mathrm{CFHO}$, the cumulative prevalence of $\mathrm{CCO}$ and conventional OA continued to increase linearly with age (Figure 2). This linear relationship between OA and $\mathrm{CCO}$ prevalence with age is supportive of previous reports in Golden and Labrador retrievers. ${ }^{4,32}$ This behavior reinforces other studies showing that laxity-based, secondary joint changes occur at any age and therefore hip dysplasia can manifest even after hip screening at 2 years of age. ${ }^{4,7}$ As in the Labrador study, the linear progression of OA is highly suggestive that many more Corgis would have developed laxity-based OA if radiographed at an older age ( $>10$ years). The oldest Corgi at 10.8 years of age showed all three radiographic signs, OA, $\mathrm{CCO}$ and $\mathrm{CFHO}$, supporting this contention. In conclusion, the evidence from this study supports that the $\mathrm{CCO}$ in the Corgi has clinical significance similar to that in large-breed dogs is a predictor for subsequent disease as well as a radiographic sign, by itself, of hip dysplasia. ${ }^{9-12,21}$

A limitation of this study is that dogs were not followed longitudinally to assess the development and progression of disease in relation to the radiographic findings. Although a majority of the Corgis had joint laxity and in addition radiographic findings commonly associated with hip dysplasia in large-breed dogs, the timing and ultimate development of the disease could not be assessed in the Corgi due to the lack of sequential follow-up 
radiographs. Identification and grading severity of these radiopaque lines may be reflective of radiographic sensitivity and possible observer subjectivity rather than disease severity. Another important limitation of the study was that no dogs with a DI below 0.30 were represented; therefore, it was not possible to fully assess the validity of the 0.30 threshold in the Corgi. Similarly, the prevalence of hip dysplasia may have been underestimated in the present study since many Corgis were younger than 2 years of age and may not have developed radiographic signs of OA at the time of examination.

In summary, the Pembroke Welsh Corgi shows a homogeneous radiographic hip joint phenotype, consisting of a high degree of joint laxity, very high occurrence of $\mathrm{CFHO}$ and frequent $\mathrm{CCO}$. Based on the strong relationship of the CFHO with both subluxation and hip dysplasia in addition to a growing base of supportive evidence from the literature to date, we propose that hypothesis 1 be rejected and hypothesis 2 be accepted. The presence of a CFHO should not be considered a 'normal' finding for the Corgi but rather a component of hip dysplasia as it is. large-breed dogs. Admittedly, additional studies are warranted to further evaluate the clinical significance of both $\mathrm{CCO}$ and CFHO. The CCO in the Corgis had similar clinical relevance to that in large-breed $\operatorname{dogs}$ as a marker of hip dysplasia and a predictor for OA. Conventionally defined OA was rarely seen in the Corgi, despite the presence of subluxation and DI in the OAsusceptible range. This may be due to the breed's low body weight or simply the vastly altered conformation of this chondrodysplastic breed. Canine hip dysplasia is not only highly prevalent in large- and giant-breed dogs, but this study suggests that this genetic weakness is also highly prevalent in dogs of smaller stature and with chondrodysplasia.

\section{References}

1. Witsberger TH, Armando Villamil J, Schultz LG, et al. Prevalence of and risk factors for hip dysplasia and cranial cruciate ligament deficiency in dogs. J Am Vet Med Assoc 2008;232:1818-1824.

2. The Orthopedic Foundation for Animals Web site. Hip dysplasia statistics. Available at: offa.org/hipstatbreed.html. Accessed May 31, 2011

3. Kealy RD, Lawler DF, Ballam JM, et al. Effects of diet restriction on life span and agerelated changes in dogs. J Am Vet Med Assoc 2002;220:1315-1320.

4. Smith GK, Paster ER, Powers MY, et al. Lifelong diet restriction and radiographic evidence of osteoarthritis of the hip joint in dogs. J Am Vet Med Assoc 2006;229:690-693.

5. Smith GK, Mayhew PD, Kapatkin AS, et al. Evaluation of risk factors for degenerative joint disease associated with hip dysplasia in German shepherd dogs, Golden retrievers, Labrador retrievers, and Rottweilers. J Am Vet Med Assoc 2001;219:1719-1724.

6. Popovitch CA, Smith GK, Gregor TP, et al. Comparison of susceptibility for hip dysplasia between Rottweilers and German shepherd dogs. J Am Vet Med Assoc 1995;206:648-650.

7. Smith GK, Lawler DF, Biery DN, et al. Chronology of hip dysplasia in a cohort of 48 Labrador retrievers followed for life. Vet Surg 2011 (accepted for publication)

8. Henricson B, Norberg I, Olsson SE. On the etiology and pathogenesis of hip dysplasia: a comparative review. J Small Anim Pract 1966;7:673-688

9. Morgan JP. Canine hip dysplasia: Significance of early bony spurring. Vet Radiol 1987;28:2-5.

10. Mayhew PD, McKelvie PJ, Biery DN, et al. Evaluation of a radiographic caudolateral curvilinear osteophyte on the femoral neck and its relationship to degenerative joint disease and distraction index in dogs. $\mathrm{J} \mathrm{Am} \mathrm{Vet} \mathrm{Med} \mathrm{Assoc}$ 2002;220:472-476.

11. Powers MY, Biery DN, Lawler DF, et al. Use of the caudolateral curvilinear osteophyte as an early marker for future development of osteoarthritis associated with hip dysplasia in dogs. J Am Vet Med Assoc 2004;225:233-237. 
12. Risler A, Klauer JM, Keuler NS, et al. Puppy line, metaphyseal sclerosis, and caudolateral curvilinear and circumferential femoral head osteophytes in early detection of canine hip dysplasia. Vet Radiol Ultrasound 2009;50:157166.

13. Szabo SD, Biery DN, Lawler DF, et al. Evaluation of a circumferential femoral head osteophyte as an early indicator of osteoarthritis characteristic of canine hip dysplasia in dogs. $J$ Am Vet Med Assoc 2007;231:889-892.

14. Tsai KL, Murphy KE. Clinical and genetic assessments of hip joint laxity in the Boykin spaniel. Canadian Journal of Veterinary Research 2006;70:148-150.

15. Langenbach A, Green P, Giger U, et al. Relationship between degenerative joint disease and hip joint laxity by use of distraction index and Norberg angle measurement in a group of cats. J Am Vet Med Assoc 1998;213:1439-1443.

16. Keller GG, Reed AL, Lattimer JC, et al. Hip dysplasia: A feline population study. Vet Radiol Ultrasound 1999;40:460-464.

17. Holt E. Hip dysplasia in a cat. J Small Anim Pract 1978;19:273-276.

18. Hayes MH, Wilson GP, Burt JK. Feline hip dysplasia. J Am Anim Hosp Assoc 1979;15:447448.

19. Corley EA. Hip dysplasia: A report from the orthopedic foundation for animals. Seminars in Veterinary Medicine and Surgery 1987;2:141151.

20. VIDAR Systems Corporation (@, Diagnostic Pro Advantage 2007, Herndon, VA, USA

21. Ackerman N. Hip dysplasia in the Afghan hound. Vet Radiol 1982;23:88-97.

22. Kealy RD, Lawler DF, Ballam JM, et al. Fiveyear longitudinal study on limited food consumption and development of osteoarthritis in coxofemoral joints in dogs. $\mathrm{J}$ Am Vet Med Assoc 1997;210:222-225.

23. LaFond E, Smith GK, Gregor TP, et al. Synovial fluid cavitation during distraction radiography of the coxofemoral joint in dogs. $J$ Am Vet Med Assoc 1997; 210:1294-1297.

24. PennHIP -Distraction Index Laxity Profile, March 2011, based on breeds with $\geq 20$ submitted dogs.

25. Smith GK, Gregor TP, Rhodes WH, et al. Coxofemoral joint laxity from distraction radiography and its contemporaneous and prospective correlation with laxity, subjective score, and evidence of degenerative joint disease from conventional hip-extended radiography in dogs. Am $J$ Vet Res 1993;54:1021-1042.

26. Priester WA, Mulvihill JJ. Canine hip dysplasia: Relative risk by sex, size, and breed, and comparative aspects. J Am Vet Med Assoc 1972;160:735-739.

27. Riser WH, Rhodes WH, Newton CD. Hip dysplasia: Theories of pathogenesis. In: Textbook of Small Animal Orthopedics. Eds. Newton C, Nunamaker D. Philadelphia: J.B. Lippincott, 1985;953-980

28. ParkerHG, VonHoldt BM, Quignon P, et al. An expressed Fgf4 retrogene is associated with breed-defining chondrodysplasia in domestic dogs. Science 2009;325:995-998.

29. Powers MY, Karbe GT, Gregor TP, et al. Relationship of official OFA hip scoring to PennHIP distraction index in a sample of 439 dogs. J Am Vet Med Assoc 2010; 237:532-541.

30. Falconer DS. Introduction to quantitative genetics. Falconer DS, editor. New York: Longman Scientific \& Technical; 1989

31. Kapatkin AS, Mayhew PD, Smith GK. Genetic control of canine hip dysplasia. Compendium 2002;24:681-687.

32. Karbe GT, Paster ER, Gregor TP, et al. Agedependent prevalence of radiographic hip osteoarthritis of canine hip dysplasia in Golden Retrievers (abstr). in Proceedings. Vet Ortho Soc 2010:1. 


\section{Curriculum Vitae}

\section{Persönliche Daten:}

Name:

Geburtsdatum:

Geburtsort:

Nationalität:

\section{Schulbildung:}

1988 - 1991

$1991-1996$

$1996-1998$

$1998-2000$

2000

\section{Studium:}

2001-2003

2003-2007

\section{Georga Tiffany Karbe}

14. Juli 1981

Boynton Beach, Florida

Deutschland/ Vereinigte Staaten
Sonnenhalde Schule, Konstanz, Deutschland

Grundschule

Pike School, Andover, Massachusettes

Grundschule, 4. bis 8. Klasse

Greenhill School, Dallas, Texas

High School 9. und 10. Klasse

Singapore American School, Singapur, Singapur

High School 11. und 12. Klasse - High School Abschluss

Abitursanerkennung vom Land Baden-Württemberg

Numerus Clausus 1,9

University of Veterinary Science, Budapest, Ungarn 07/2003 Physikumsprüfung

Freie Universität Berlin, Berlin, Deutschland 01/2007 Approbation als Tierärztin (med. vet.)

\section{Anfertigung der Dissertation:}

09/2009- dato

\section{Berufserfahrung:}

03/2007- 06/2007

2007-2008

2008- 2010

2010- 2011

2011- dato unter der Leitung von Prof. Dr. Urs Giger am Department für Kleintiere, der Vetsuisse-Fakultät Universität Zürich

Direktorin: Prof. Dr. Claudia Reusch

Institut für Veterinär-Anatomie, Freie Universität Berlin Lehrangestellte

Colorado State University, Fort Collins, Colorado Internship Kleintierchirurgie und innere Medizin

University of Pennsylvania, Philadelphia, Pennsylvania PennHIP Orthopaedic Research Fellowship, Doktorandin

University of Pennsylvania, Philadelphia, Pennsylvania Internship Kleintierchirurgie

University of Pennsylvania, Philadelphia, Pennsylvania Residency Kleintierchirurgie 


\section{Acknowledgements}

I thank first and foremost my advisors, Profs. Urs Giger and Gail Smith, for making this dissertation possible. I am grateful for their continued guidance and support throughout this project. It has truly been a pleasure working with and learning from such renowned experts and mentors.

I would also like to sincerely thank Prof. Darryl Biery for evaluating all the hip radiographs and for teaching me the intricacies of radiographic interpretation of the canine hip. Furthermore, I thank Thomas Gregor for all the statistical support and data analysis.

A special thank you is dedicated to Christine Paris, Dr. Pamela McKelvie and the PennHIP team for their help and encouragement.

From the bottom of my heart I want to thank my fiance, Thomas Knorpp, and my dear family, Hartwig, Monika and Antonia Karbe, for all your support and ecouragement throughout the years, I could not have done it without you. 